\title{
SQUARES IN QUADRATIC PROGRESSION
}

\author{
R. G. E. PINCH
}

\begin{abstract}
The sequence of consecutive integer squares has constant second difference 2 . We list every such sequence of squares containing a term less than $1000^{2}$.
\end{abstract}

\section{INTRODUCTION}

We call a sequence a quadratic progression if the second difference is constant. The sequence of consecutive integer squares is certainly a quadratic progression with second difference equal to 2 . A number of families of nontrivial fourterm integer square progressions with second difference 2 are known (Buell [2]) but no such five-term quadratic progression of integer squares is known. Leech asked whether there is such a sequence starting at 0 and we answer this in the negative by listing all four-term progressions with an element less than 1000 and showing that none includes 0 . We also find that none of these progressions can be extended to a fifth square term.

\section{QUADRATIC PROGRESSIONS WITH GIVEN SECOND TERM}

Suppose that

$$
\begin{array}{ll}
x^{2}, \quad & d^{2}, \quad y^{2}=2 d^{2}-x^{2}+2, \\
& z^{2}=3 d^{2}-2 x^{2}+6
\end{array}
$$

is a four-term progression with constant second difference 2 . We regard the second term $d^{2}$ as given. Then we have

$$
\begin{aligned}
y^{2}+x^{2} & =2 d^{2}+2, \\
z^{2}+2 x^{2} & =3 d^{2}+6
\end{aligned}
$$

with obvious solutions $\pm x=d \pm 1$ corresponding to the trivial quadratic progression of consecutive squares. For given $d$, it is easy to tabulate the finite set of solutions $x$ to each of the individual equations in this pair of equations. The results for $d \leq 999$ are included in Table 1 .

Received by the editor November 20, 1990 and, in revised form, July 17, 1992.

1991 Mathematics Subject Classification. Primary 11B83; Secondary 11D25, 11 Y50.

(C) 1993 American Mathematical Society $0025-5718 / 93 \$ 1.00+\$ .25$ per page 
TABLE 1. Table of solutions

\begin{tabular}{|c|c|c|c|c|c|c|c|}
\hline$t$ & $x$ & $y$ & $z$ & $t$ & $x$ & $y$ & $z$ \\
\hline 6 & 23 & 32 & 39 & 570 & 7879 & 11128 & 13623 \\
\hline 16 & 87 & 122 & 149 & 584 & 2257 & 3138 & 3821 \\
\hline 39 & 32 & 23 & 6 & 601 & 4832 & 6807 & 8326 \\
\hline 39 & 70 & 91 & 108 & 651 & 580 & 499 & 402 \\
\hline 51 & 148 & 203 & 246 & 651 & 778 & 887 & 984 \\
\hline 59 & 228 & 317 & 386 & 849 & 718 & 557 & 324 \\
\hline 59 & 630 & 889 & 1088 & 856 & 1537 & 1998 & 2371 \\
\hline 79 & 242 & 333 & 404 & 883 & 25566 & 36145 & 44264 \\
\hline 83 & 516 & 725 & 886 & 862 & 1713 & 2264 & 2705 \\
\hline 108 & 91 & 70 & 39 & 886 & 725 & 516 & 83 \\
\hline 108 & 157 & 194 & 225 & 916 & 26605 & 37614 & 46063 \\
\hline 108 & 707 & 994 & 1215 & 984 & 887 & 778 & 651 \\
\hline 108 & 6643 & 9394 & 11505 & 984 & 1145 & 1286 & 1413 \\
\hline 147 & 302 & 401 & 480 & 1088 & 889 & 630 & 59 \\
\hline 149 & 122 & 87 & 16 & 1215 & 994 & 707 & 108 \\
\hline 177 & 878 & 1229 & 1500 & 1226 & 1017 & 752 & 311 \\
\hline 225 & 194 & 157 & 108 & 1247 & 1048 & 801 & 430 \\
\hline 225 & 296 & 353 & 402 & 1411 & 1180 & 891 & 442 \\
\hline 240 & 839 & 1162 & 1413 & 1413 & 1162 & 839 & 240 \\
\hline 246 & 203 & 148 & 51 & 1413 & 1286 & 1145 & 984 \\
\hline 287 & 11838 & 16739 & 20500 & 1500 & 1229 & 878 & 177 \\
\hline 311 & 752 & 1017 & 1226 & 2371 & 1998 & 1537 & 856 \\
\hline 324 & 557 & 718 & 849 & 2561 & 2112 & 1537 & 514 \\
\hline 334 & 3693 & 5212 & 6379 & 2705 & 2264 & 1713 & 862 \\
\hline 386 & 317 & 228 & 59 & 2853 & 2348 & 1699 & 510 \\
\hline 386 & 6237 & 8812 & 10789 & 3821 & 3138 & 2257 & 584 \\
\hline 402 & 353 & 296 & 225 & 6150 & 5029 & 3572 & 477 \\
\hline 402 & 499 & 580 & 651 & 6379 & 5212 & 3693 & 334 \\
\hline 404 & 333 & 242 & 79 & 8326 & 6807 & 4832 & 601 \\
\hline 419 & 11020 & 15579 & 19078 & 10789 & 8812 & 6237 & 386 \\
\hline 430 & 801 & 1048 & 1247 & 11505 & 9394 & 6643 & 108 \\
\hline 442 & 891 & 1180 & 1411 & 13623 & 11128 & 7879 & 570 \\
\hline 477 & 3572 & 5029 & 6150 & 19078 & 15579 & 11020 & 419 \\
\hline 480 & 401 & 302 & 147 & 20500 & 16739 & 11838 & 287 \\
\hline 510 & 1699 & 2348 & 2853 & 44264 & 36145 & 25566 & 883 \\
\hline 514 & 1537 & 2112 & 2561 & 46063 & 37614 & 26605 & 916 \\
\hline
\end{tabular}




\section{QUADRATIC PROGRESSIONS WITH GIVEN FIRST TERM}

Suppose that

$$
c^{2}, \quad x^{2}, \quad y^{2}=2 x^{2}-c^{2}+2, \quad z^{2}=3 x^{2}-2 c^{2}+6
$$

is a four-term quadratic progression with constant second difference 2 . We regard the first term $c^{2}$ as given. Then we have a pair of simultaneous Pellian equations

$$
y^{2}-2 x^{2}=2-c^{2}, \quad z^{2}-3 x^{2}=6-2 c^{2}
$$

with obvious solutions $\pm x=c \pm 1$ corresponding to the trivial quadratic progression of consecutive squares. For given $c$, each Pellian equation has infinitely many solutions $x$. We shall show that in practice a simple search can be used to identify small common solutions, and that Baker's method of linear forms in logarithms can be used to determine whether or not the set of solutions found in this way is complete.

We illustrate the method on the case $c=39$, which exhibits all the features of interest. We have

$$
y^{2}-2 x^{2}=-1519, \quad z^{2}-3 x^{2}=-3036 .
$$

The first equation is solved by working in the field $\mathbb{Q}(\sqrt{2})$, which has class number 1 and fundamental unit $1+\sqrt{2}$. We have

$$
\pm y \pm x \sqrt{2}=\delta_{i} \eta^{n}
$$

for some integer $n$ and $i=1,2$ or 3 , where $\eta=3+2 \sqrt{2}$ is the fundamental totally positive unit and $\delta_{1}=7+28 \sqrt{2}, \delta_{2}=23+32 \sqrt{2}, \delta_{3}=37+38 \sqrt{2}$. The possible values of $\pm x$ therefore fall into three binary recurrence sequences $A_{n}^{(i)}$, each with recurrence relation

$$
A_{n+1}=6 A_{n}-A_{n-1},
$$

which has the minimal equation for $\eta$ as auxiliary polynomial, and initial values $A_{0}^{(1)}=28, A_{1}^{(1)}=98 ; A_{0}^{(2)}=32, A_{1}^{(2)}=142 ; A_{0}^{(3)}=38, A_{1}^{(3)}=188$, respectively.

We solve the second equation similarly in $\mathbb{Q}(\sqrt{3})$, which has class number 1 and fundamental unit $2+\sqrt{3}$. We have

$$
\pm z \pm x \sqrt{3}=\beta_{i} \varepsilon^{m}
$$

for some integer $m$ and $j=1$ or 2 , where $\varepsilon=2+\sqrt{3}$ is the fundamental totally positive unit and $\beta_{1}=6+32 \sqrt{3}, \beta_{2}=36+38 \sqrt{3}$. The possible values of $\pm x$ therefore fall into two binary recurrence sequences $B_{m}^{(j)}$, each with recurrence relation

$$
B_{m+1}=4 B_{m}-B_{m-1},
$$

which has the minimal equation for $\varepsilon$ as auxiliary polynomial, and initial values $B_{0}^{(1)}=32, B_{1}^{(1)}=70 ; B_{0}^{(2)}=38, B_{1}^{(2)}=112$, respectively.

The solutions $x$ to the original pair of equations are therefore the common values between the sequences $A_{n}^{(i)}$ and $B_{m}^{(j)}$. Computing the values of the sequences with $m, n$ from -10 to +10 , we find that $A_{-1}^{(1)}=B_{1}^{(1)}=70$, $A_{0}^{(2)}=B_{0}^{(1)}=32, A_{0}^{(3)}=B_{0}^{(2)}=38$, and $A_{-1}^{(3)}=B_{-1}^{(2)}=40$. We shall show that 
these are in fact the only occurrences of common values between the sequences $A^{(i)}$ and $B^{(j)}$.

We show first that there is no term in common between the pairs of sequences $A^{(1)}$ and $B^{(2)}$, between $A^{(2)}$ and $B^{(2)}$, or between $A^{(3)}$ and $B^{(1)}$. We do so by considering the (finite) sets of values of $A^{(1)}$ and $B^{(2)}$ modulo $41, A^{(2)}$ and $B^{(2)}$ modulo $408, A^{(3)}$ and $B^{(1)}$ modulo 315 , and observing that in each case the sets of $A$-values and $B$-values are disjoint.

We now show that the only values in common between the pairs of sequences $A^{(1)}$ and $B^{(1)}$; between $A^{(2)}$ and $B^{(1)}$; and between $A^{(3)}$ and $B^{(2)}$ are the values given above. By Theorem 2.6 of [3] any solution to $y+x \sqrt{2}=\delta_{1} \eta^{n}$, $z+x \sqrt{3}=\beta_{1} \varepsilon^{m}$, must have $m, n$ less than $\exp (63.11)$. We apply Algorithm 3.1 of [3] to the sequences $A^{(1)}$ and $B^{(1)}$ with List $\mathrm{K}$ the set of primes up to 953 and List $\mathrm{L}$ the primes less than 241 and find that if $A_{n}^{(1)}=B_{m}^{(1)}$, then $n \equiv-1 \bmod N$ and $m \equiv 1 \bmod M$ for moduli $N, M$ with $\log N \geq 79.05$ and $\log M \geq 100.00$, which shows that in fact we must have $n=-1$ and $m=1$ as required. A similar analysis holds for the pair of sequences $A^{(2)}$ and $B^{(1)}$.

To show that the sequences $A^{(3)}$ and $B^{(2)}$ have only the two values 38 and 40 in common, we split $A^{(3)}$ up into two subsequences $C^{(1)}$ and $C^{(2)}$ of alternate terms, having the recurrence relations $C_{n+1}=34 C_{n}-C_{n-1}$ with initial values $C_{0}^{(1)}=A_{0}^{(3)}=38, C_{1}^{(1)}=A_{2}^{(3)}=1090 ; C_{0}^{(2)}=A_{-1}^{(3)}=40$, $C_{1}^{(2)}=A_{1}^{(3)}=188$. Again computing the values of $C_{n}^{(i)}$ for $i$ between -10 and +10 , we find that the only common values are $C_{0}^{(1)}=B_{0}^{(2)}=38$ and $C_{0}^{(2)}=B_{-1}^{(2)}=40$. We now apply Algorithm 3.1 of [3] to each pair of sequences in turn as before to show that these are indeed the unique common values.

Theorem 1. The only nontrivial four-term quadratic progressions of integer squares with a term less than $1000^{2}$ are those given by Table 1 or their reversal. Proof. Since reversing a quadratic progression with constant second difference 2 gives another such, it is sufficient to consider those for which the first or the second term is less than 1000 . Those for which the second term is less than 1000 are dealt with by the remarks of $\S 1$. The calculation illustrated above for the case $c=39$ is simple to automate and was programmed in Algol-68C. In each case where sequences appeared to have no common term, it was possible to show this by taking values to a modulus at most 965 . In each case where sequences had a term in common, the bound on the exponents $m$ and $n$ was less than $\exp (63.11)$, and the algorithm was applied with List $\mathrm{K}$ being the primes up to 953 and List $L$ the primes up to 241 . Each application of the algorithm produced moduli in excess of the bound, showing that each pair of sequences with apparently only one term in common did indeed have only that term as a common value. The whole table was computed and verified completely on a Sun $3 / 60$ workstation, in about eleven hours of CPU time.

\section{OTHER SECOND DIFFERENCES}

Similar results can be obtained for any fixed constant second difference $\delta$. We replace (1.2) by

$$
y^{2}+x^{2}=2 d^{2}+\delta, \quad z^{2}+2 x^{2}=3 d^{2}+3 \delta,
$$


and (2.2) by

$$
y^{2}-2 x^{2}=\delta-c^{2}, \quad z^{2}-3 x^{2}=3 \delta-2 c^{2},
$$

and it is clear that the same techniques apply.

The problem of finding squares in quadratic progression with constant but unrestricted second difference is equivalent to finding a quadratic polynomial with consecutive square values. This problem was considered by Allison [1], who obtains the sequence $53^{2}, 173^{2}, 217^{2}, 233^{2}, 227^{2}, 197^{2}, 127^{2}$ of seven distinct squares with constant second difference -9960 . He shows that there are infinitely many quadratic polynomials taking eight consecutive square values symmetric about the turning point if this falls midway between integers, the first giving the progression

$$
17^{2}, 53^{2}, 67^{2}, 73^{2}, 73^{2}, 67^{2}, 53^{2}, 17^{2}
$$

with constant second difference -840 . In [4] it is shown that there are no such polynomials if the turning point is at an integer. In each of these "symmetric" cases the argument reduces to analyzing the group of rational points on an elliptic curve given by simultaneous Pell equations.

\section{BIBLIOGRAPHY}

1. Allison, On square values of quadratics, Math. Proc. Cambridge Philos. Soc. 99 (1986), 381-383.

2. D. Buell, Integer squares with constant second difference, Math. Comp. 49 (1987), 635-644.

3. R. G. E. Pinch, Simultaneous Pellian equations, Math. Proc. Cambridge Philos. Soc. 103 (1988), 35-46.

4. __ Square values of quadratic polynomials, (to appear).

Department of Pure Mathematics and Mathematical Statistics, University of CamBRIdGe, 16 Mill LANE, CAMbridge CB2 1SB, UNited Kingdom

E-mail address: rgep@phx.cam.ac.uk 